\title{
Chapter 2 \\ Bond Graphs: A Unifying Framework for Modelling of Physical Systems
}

\author{
Jan F. Broenink
}

\begin{abstract}
This chapter introduces a formalism to model the dynamic behaviour of physical systems known as bond graphs. A important property of this formalism is that systems from different domains (cf. electrical, mechanical, hydraulical, acoustical, thermodynamical, material) are described in the same way an integrated under the unifying concept of energy exchange. Bond graph models are directed graphs where parts are interconnected by bonds, along which exchange of energy occurs. We present a method to systematically build a bond graph starting from an ideal physical model and present methods to perform the causal analysis of bond graphs and procedures to generate equations to enable simulation.
\end{abstract}

\section{Learning Objectives}

After reading this chapter, we expect you to be able to:

- Use bond graphs as an abstraction to model bi-directional energy exchange between components in a domain-neutral fashion

- Be able to translate domain dependent diagrams into ideal physical models and subsequently into bond-graph models

- Translate bond graph models into systems of differential equations for simulation and analysis

\subsection{Introduction}

Bond graphs are a domain-independent graphical description of dynamic behaviour of physical systems. This means that systems from different domains (cf. electrical, mechanical, hydraulical, acoustical, thermodynamical, material) are described in the same way. The basis is that bond graphs are based on energy and energy exchange. Analogies between domains are more than just equations being analogous: the used physical concepts are analogous.

Bond-graph modelling is a powerful tool for modelling engineering systems, especially when different physical domains are involved. Furthermore, bond-graph submodels can be re-used elegantly, because bondgraph models are non-causal. The submodels can be seen as objects; bond-graph modelling is a form of object-oriented physical systems modelling.

Bond graphs are labelled and directed graphs, in which the vertices represent submodels and the edges represent an ideal energy connection between power ports. The vertices are idealised descriptions of physical phenomena: it are concepts, denoting the relevant (i.e. dominant and interesting) aspects of the dynamic behaviour of the system. It can be bond graphs itself, thus allowing hierarchical models, or it can be a set of

Jan F. Broenink

University of Twente, Netherlands

e-mail: j.f.broenink@utwente.nl 
equations in the variables of the ports (two at each port). The edges are called bonds. They denote point-to-point connections between submodel ports.

When preparing for simulation, the bonds are embodied as two-signal connections with opposite directions. Furthermore, a bond has a power direction and a computational causality direction. Proper assigning the power direction resolves the sign-placing problem when connecting submodels structures. The internals of the submodels give preferences to the computational direction of the bonds to be connected. The eventually assigned computational causality dictates which port variable will be computed as a result (output) and consequently, the other port variable will be the cause (input). Therefore, it is necessary to rewrite equations if another computational form is specified then is needed. Since bond graphs can be mixed with block-diagram parts, bond-graph submodels can have power ports, signal inputs and signal outputs as their interfacing elements. Furthermore, aspects like the physical domain of a bond (energy flow) can be used to support the modelling process.

The concept of bond graphs was originated by [228]. The idea was further developed by Karnopp and Rosenberg in their textbooks $([161,162,160])$, such that it could be used in practice $[268,86]$. By means of the formulation by Breedveld [43, 44] of a framework based on thermodynamics, bond-graph model descriptions evolved to a systems theory.

In the next section, we will introduce the bond graph method by some examples, where we start from a given network composed of ideal physical models. Transformation to a bond graph leads to a domain independent model. In Section 3, we will introduce the foundations of bond graphs, and present the basic bond graph elements in Section 4. We will discuss a systematic method for deriving bond graphs from engineering systems in Section 5. How to enhance bond-graph models to generate the model equations and for analysis is presented in Section 6 , and is called Causal Analysis. The equations generation and block diagram expansion of causal bond graphs is treated in Sections 7 and 8. Section 9 discusses simulation issues. In Section 10 we review this chapter, and also include some hints for further reading.

\subsection{Bond-Graph Examples}

To introduce bond graphs, we will discuss examples of two different physical domains, namely an RLC circuit (electrical domain) and a damped mass-spring system (mechanical domain, translation). The RLC circuit is given in Figure 2.1.



Fig. 2.1: The RLC cicuit

In electrical networks, the port variables of the bond-graph elements are the electrical voltage over the element port and electrical current through the element port. Note that a port is an interface of an element to other elements; it is the connection point of the bonds. The power being exchanged by a port with the rest of the system is the product of voltage and current: $P=u i$. The equations of a resistor, capacitor and inductor are: 


$$
\begin{aligned}
& u_{R}=i R \\
& u_{C}=\frac{1}{C} \int i \mathrm{~d} t \\
& i_{L}=\frac{1}{I} \int u \mathrm{~d} t
\end{aligned}
$$

In order to facilitate the conversion to bond graphs, we draw the different elements of the electric domain in such a way that their ports become visible. For brevity, we only show this for the Capacitor Figure 2.2. To this port, we connect a power bond or bond for short. This bond denotes the energy exchange between the elements. A bond is drawn as an edge with half an arrow. The direction of this half arrow denotes the positive direction of the energy flow. In principle, the voltage source delivers power and the other elements absorb power.
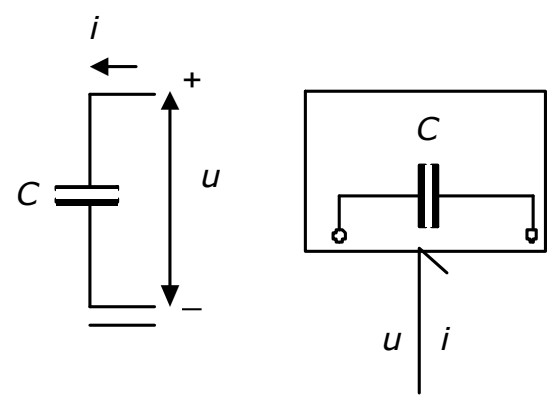

Fig. 2.2: Electric Capacitor: Circuit (left) and with bond (right)

Considering the circuit of Figure 2.1, we see that the voltage over the elements are different and through all elements flows the same current. We indicate this current with $i$ and connect the bonds of all elements with this current $i$ (2.3). Changing the electric symbols into corresponding bond graph mnemonics, result in the bond graph of the electrical circuit. The common $i$ is changed to a '1', a so-called 1-junction. Writing the specific variables along the bonds makes the bond graph an electric bond graph. The voltage is mapped onto the domain-independent effort variable and the current maps onto the domain-independent flow variable (the current always on the side of the arrow). The 1-junction means that the current (flow) through all connected bonds is the same, and that the voltages (efforts) sum to zero, considering the sign. This sign is related to the power direction (i.e. direction of the half arrow) of the bond. This summing equation is the Kirchhoff voltage law.

Parallel connections, in which the voltage over all connected elements is the same, are denoted by a $u$ in the port-symbol network. The bond-graph mnemonic is a 0 , the so-called 0 -junction. A 0 -junction means that the voltage (effort) over all connected bonds is the same, and that the currents (flows) sum to zero, considering the sign. This summing equation is the Kirchhoff current law.

The second example is the damped mass-spring system, a mechanical system shown in 2.4. In mechanical diagrams, the port variables of the bond graph elements are the force on the element port and velocity of the element port. For the rotational mechanical domain, the port variables are the torque and angular velocity. Again, two variables are involved. The power being exchanged by a port with the rest of the system is the product of force and velocity: $P=F v(P=T \omega$ for the rotational case). The equations of a damper, spring and mass are (we use damping coefficient $a$, spring coefficient $K_{s}$, mass $\mathrm{m}$ and applied force $F_{a}$ ):

$$
\begin{aligned}
F_{d} & =\alpha v \\
F_{s} & =K_{s} \int v \mathrm{~d} t=\frac{1}{C_{s}} \int v \mathrm{~d} t \\
F_{m} & =m \frac{d v}{d t} \quad \text { or } \quad v=\frac{1}{m} \int F_{m} \mathrm{~d} t \\
F_{a} & =\text { force }
\end{aligned}
$$





Fig. 2.3: Bond graph with electrical symbols (left) and with standard symbols (right). The standard bond-graph symbols are defined in 2.4

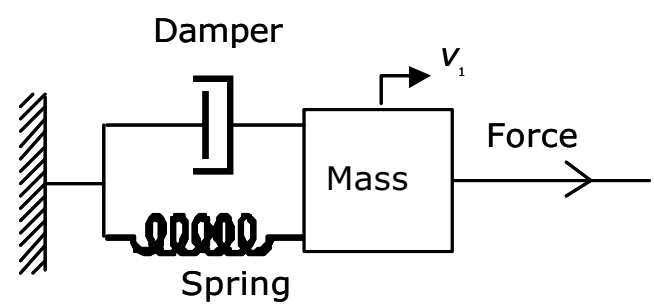

Fig. 2.4: The damped mass-spring system

In the same way as with the electrical circuit, we can redraw the element such that their ports become visible (2.5). The loose ends of the example all have the same velocity, which is indicated by a $v$. This junction element also implies that the forces sum up to zero, considering the sign (related to the power direction). The force is mapped onto an effort and the velocity onto a flow. For the rotational mechanical domain, the torque is mapped onto an effort and the angular velocity onto a flow. This implies that force is related to electric voltage and that velocity is related to electric current.

We see the following analogies between the mechanical and electrical elements:

- The damper is analogous to the resistor.

- The spring is analogous to the capacitor; the mechanical compliance corresponds with the electrical capacity.

- The mass is analogous to the inductor.

- The force source is analogous to the voltage source.

- The common velocity is analogous to the loop current.

Besides points with common velocity, also points with common force exist in mechanical systems. Then forces are all equal and velocities sum up to zero, considering the sign (related to the power direction). These common force points are denoted as 0 -junctions in a bond graph (an example is a concatenation of a mass, a spring and a damper: the three elements are connected in 'series'). A further elaboration on analogies can be found in the next section, where the foundations of bond graphs are discussed.

Through these two examples, we have introduced most bond graph symbols and indicated how in two physical domains the elements are transformed into bond graph mnemonics. One group of bond graph elements was not yet introduced: namely the transducers. Examples are the electric transformer, an electric motor and toothed wheels. In the next section, we will discuss the foundations of bond graphs. 

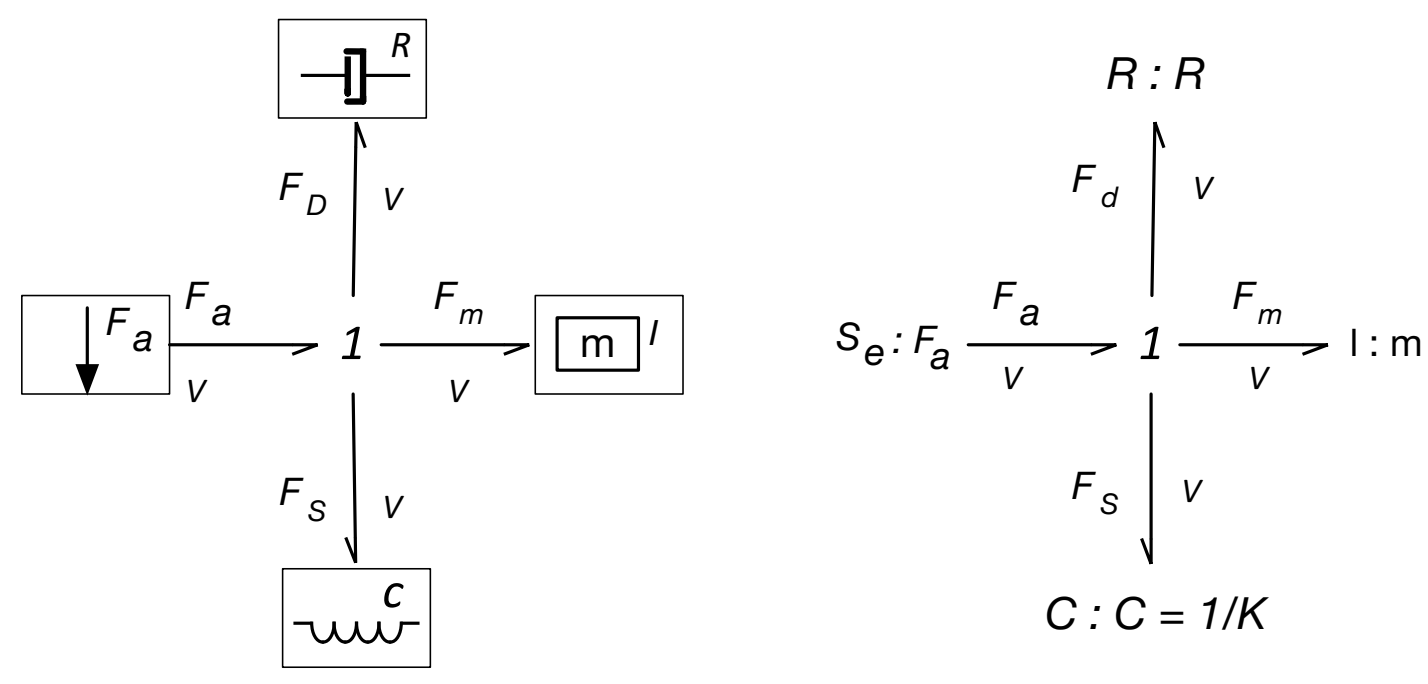

Fig. 2.5: Bond graph with mechanical symbols (left) and with standard symbols (right)

\subsection{Foundation of Bond Graphs}

Analogies between different systems were shown in the previous section: Different systems can be represented by the same set of differential equations. These analogies have a physical foundation: the underlying physical concepts are analogous, and consequently, the resulting differential equations are analogous. The physical concepts are based on energy and energy exchange. Behaviour with respect to energy is domain independent. It is the same in all engineering disciplines, as can be concluded when comparing the RLC circuit with the damped mass spring system. This leads to identical bond graphs.

\subsubsection{Starting Points}

Before discussing the specific properties of bond graphs and the elementary physical concepts, we first recall the assumptions general for network like descriptions of physical systems, like electrical networks, mechanical or hydraulic diagrams:

- The conservation law of energy is applicable.

- It is possible to use a lumped approach.

This implies that it is possible to separate system properties from each other and to denote them distinctly, while the connections between these submodels are ideal. Separate system properties mean physical concepts and the ideal connections represent the energy flow, i.e. the bonds between the submodels. This idealness property of the connections means that in these connections no energy can be generated or dissipated. This is called power continuity. This structure of connections is a conceptual structure, which does not necessary have a size. This concept is called reticulation [228] or tearing [173]. See also [304].

The system's submodels are concepts, idealised descriptions of physical phenomena, which are recognised as the dominating behaviour in components (i.e. real-life, tangible system parts). This implies that a model of a concrete part is not necessary only one concept, but can consist of a set of interconnected concepts. 


\subsubsection{Bonds and Ports}

The contact point of a submodel where an ideal connection will be connected to is called a power port or port for short. The connection between two submodels is called a power bond or bond; it is drawn as a single line (2.6). This bond denotes an ideal energy flow between the two connected submodels. The energy entering the bond on one side immediately leaves the bond at the other side (power continuity).

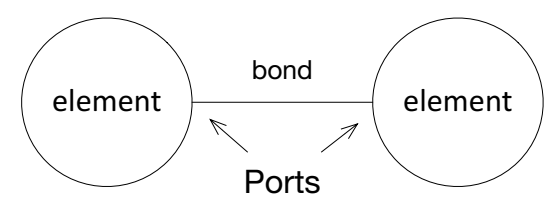

Fig. 2.6: The energy flow between two submodels represented by a bond.

The energy flow along a bond has the physical dimension of power, being the product of two variables. In each physical domain, there is such a combination of variables, for which a physical interpretation is useful. In electrical networks, the two variables are voltage and current. In mechanical systems, the variable pairs are force and velocity for translation and torque and angular velocity for rotation. In hydraulics, it is pressure and volume flow. For thermodynamic systems, temperature and entropy flow are used. These pairs of variables are called (power-) conjugated variables.

In order to understand the connection as established by a bond, this bond can be interpreted in two different ways, namely:

1. As an interaction of energy.

The connected subsystems form a load to each other by their energy exchange. A power bond embodies a connection where a physical quantity is exchanged.

2. As a bilateral signal flow.

The connection is interpreted as two signals, an effort and flow, flowing in opposite direction, thus determining the computational direction of the bond variables. With respect to one of the connected submodels, the effort is the input and the flow the output, while for the other submodel input and output are of course established by the flow and effort respectively.

These two ways of conceiving a bond is essential in bond graph modelling. Modelling is started by indicating the physical structure of the system. The bonds are first interpreted as interactions of energy, and then the bonds are endowed with the computational direction, interpreting the bonds as bilateral signal flows. During modelling, it need not be decided yet what the computational direction of the bond variables is. Note that, determining the computational direction during modelling restricts submodel reuse. It is however necessary to derive the mathematical model (set of differential equations) from the graph. The process of determining the computational direction of the bond variables is called causal analysis. The result is indicated in the graph by the so-called causal stroke, indicating the direction of the effort, and is called the causality of the bond (2.7).

In equation form, 2.7 can be written as:

$$
\begin{aligned}
& \text { element } 1 . e:=\text { element } 2 . e \\
& \text { element } 2 . f:=\text { element } 1 . f
\end{aligned}
$$

$$
\begin{array}{r}
\text { element } 2 . e:=\text { element } 1 . e \\
\text { element } 1 . f:=\text { element } 2 . f
\end{array}
$$

\subsection{Bond-Graph Elements}

The constitutive equations of the bond graph elements are introduced via examples from the electrical and mechanical domains. The nature of the constitutive equations lay demands on the causality of the connected bonds. Bond graph elements are drawn as letter combinations (mnemonic codes) indicating the type of element. The bond graph elements are the following: 


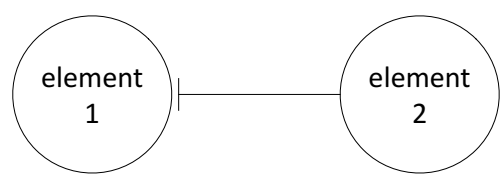

$=$

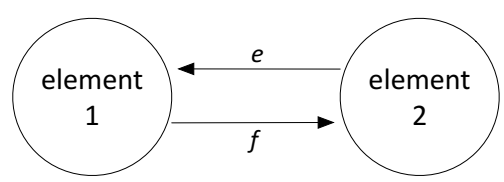

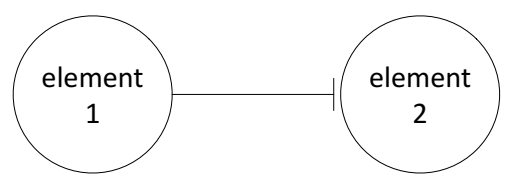

$=$



Fig. 2.7: Determine the signal direction of the effort and flow (we do not use the power direction at the bonds, so it is not shown here)

C storage element for a q-type variable, e.g. capacitor (stores charge), spring (stores displacement).

I storage element for a p-type variable, e.g. inductor (stores flux linkage), mass (stores momentum).

R Resistor dissipating free energy, e.g. electric resistor, mechanical friction.

Se, Sf sources, e.g. electric mains (voltage source), gravity (force source), pump (flow source).

TF transformer, e.g. an electric transformer, toothed wheels, lever.

GY gyrator, e.g. electromotor, centrifugal pump.

$\mathbf{0 , 1} 0$ - and 1 -junctions, for ideal connecting two or more submodels.

\subsubsection{Storage Elements}

Storage elements store all kinds of free energy. As indicated above, there are two types of storage elements: $\mathrm{C}$-elements and I-elements. The $q$-type and $p$-type variables are conserved quantities and are the result of an accumulation (or integration) process. They are the (continuous) state variables of the system.

In C-elements, like a capacitor or spring, the conserved quantity, $q$, is stored by accumulating the net flow, $f$, to the storage element. This results in the differential equation:

$$
\dot{q}=f
$$

which is called a balance equation, and forms a part of the constitutive equations of the storage element. In the other part of the constitutive equations, the state variable, $q$, is related to the effort, $e$ :

$$
e=e(q)
$$

This relation depends on the specific shape of the particular storage element.

In 2.8, examples of $\mathrm{C}$-elements are given together with the equivalent block diagram. The equations for a linear capacitor and linear spring are:

$$
\begin{aligned}
\dot{q} & =i, & & u=\frac{1}{C} q \\
\dot{x} & =v, & F & =K x=\frac{1}{C} x
\end{aligned}
$$

For a capacitor, $\mathrm{C}[\mathrm{F}]$ is the capacitance and for a spring, $\mathrm{K}[\mathrm{N} / \mathrm{m}]$ is the stiffness and $\mathrm{C}[\mathrm{m} / \mathrm{N}]$ the compliance. For all other domains, a C-element can be defined.

The effort variable is equal when two C-storage elements connected in parallel with a resistor in between are in equilibrium. Therefore, the domain-independent property of an effort is determination of equilibrium.

In I-elements, like a inductor or mass, the conserved quantity, $p$, is stored by accumulating the net flow, $e$, to the storage element. This results in the differential equation: 


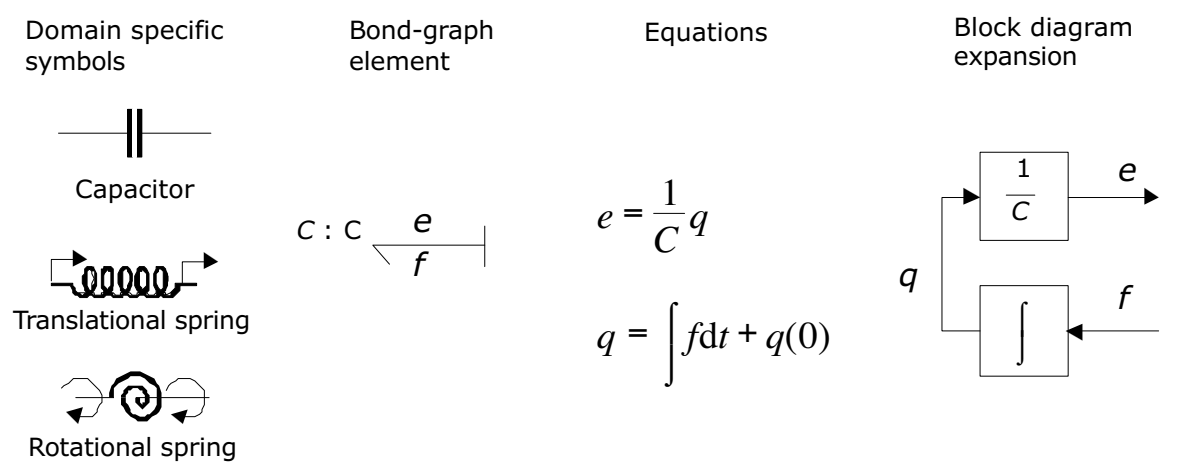

Fig. 2.8: Examples of C-elements

$$
\dot{p}=e
$$

which is called a balance equation, and forms a part of the constitutive equations of the storage element. In the other part of the constitutive equations, the state variable, $q$, is related to the effort, $e$ :

$$
f=f(q)
$$

This relation depends on the specific shape of the particular storage element.

In 2.9, examples of I-elements are given together with the equivalent block diagram. The equations for a linear inductor and linear mass are:

$$
\begin{aligned}
& \dot{\lambda}=u, i=\frac{1}{L} \lambda \\
& \dot{p}=F, v=\frac{1}{m} p
\end{aligned}
$$

For an inductor, $\mathrm{L}[\mathrm{H}]$ is the inductance and for a mass, $\mathrm{m}[\mathrm{kg}]$ is the mass. For all other domains, an I-element can be defined.

The flow variable is equal when two I-storage elements connected in parallel with a resistor in between, are in equilibrium. Therefore, at I-elements, the domain-independent property of the flow is determination of equilibrium. F or example, when two bodies, moving freely in space each having a different momentum, are being coupled (collide and stick together), the momentum will divide among the masses such that the velocity of both masses is the same (this is the conservation law of momentum).

Note that when at the two types of storage elements, the role of effort and flow are exchanged: the C- element and the I-element are each other's dual form.

The block diagrams in 2.8 and 2.9, and also in the next Figures 10 to 16, show the computational direction of the signals involved. They are indeed the expansion of the corresponding causal bond graph. The equations are given in computational form, consistent with the causal bond graph and the block diagram.

\subsubsection{Resistors}

Resistors, R-elements, dissipate free energy. Examples are dampers, frictions and electric resistors (2.10). In real-life mechanical components, friction is always present. Energy from an arbitrary domain flows irreversibly to the thermal domain (and heat is produced). This means that the energy flow towards the resistor is always 


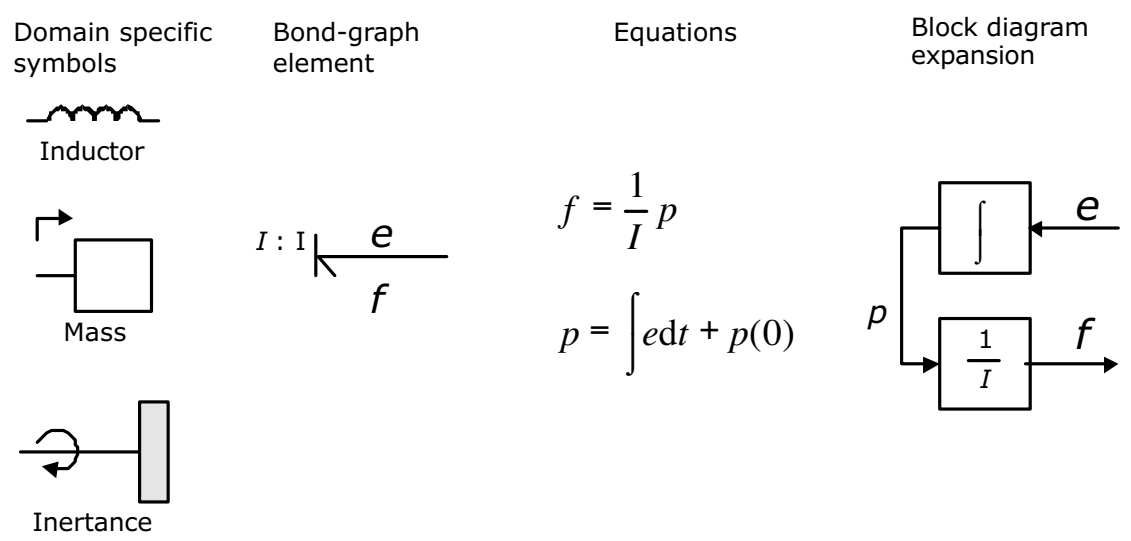

Fig. 2.9: Examples of I-elements

positive. The constitutive equation is an algebraic relation between the effort and flow, and lies principally in the first or third quadrant.

$$
e=f(f)
$$

Domain-specific symbols

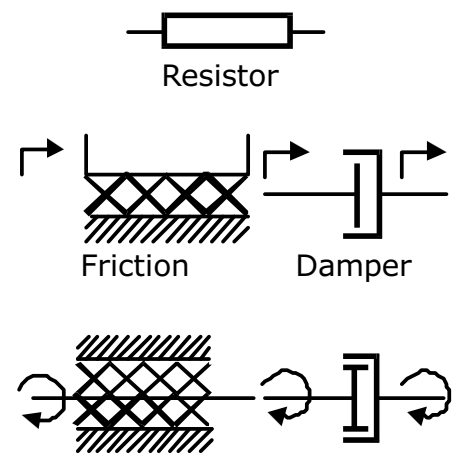

Bond-graph element

$R: \mathrm{R}$

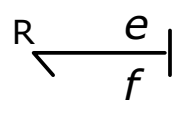

$R: \mathrm{R} \frac{e}{f}$
Equations

$e=R f$

$f=\frac{1}{R} e$
Block diagram expansion

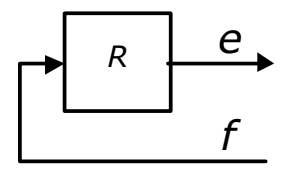

e



Fig. 2.10: Examples of resistors

An electrical resistor is mostly linear (at constant temperature), namely Ohm's law. The electrical resistance value is in $[\Omega]$.

$$
u=R i
$$

Mechanical friction mostly is non-linear. The resistance function is a combination of dry friction and viscous friction. Dry friction is a constant friction force and viscous friction is the linear term. Sometimes, also stiction 
is involved, a tearing-loose force only applicable when starting a movement. All these forms of friction can be modelled with the R-element. The viscous friction has as formula $(R$ in $[\mathrm{Ns} / \mathrm{m}]$ :

$$
F=R v
$$

If the resistance value can be controlled by an external signal, the resistor is a modulated resistor, with mnemonic MR. An example is a hydraulic tap: the position of the tap is controlled from the outside, and it determines the value of the resistance parameter.

If the thermal domain is modelled explicitly, the production of thermal energy should explicitly be indicated. Since the dissipator irreversibly produces thermal energy, the thermal port is drawn as a kind of source of thermal energy. The R becomes an RS.

\subsubsection{Sources}

Sources represent the interaction of a system with its environment. Examples are external forces, voltage and current sources, ideal motors, etc. (2.11). Depending on the type of the imposed variable, these elements are drawn as Se or Sf.

Besides as a 'real' source, source elements are used to give a variable a fixed value, for example, in case of a point in a mechanical system with a fixed position, a Sf with value 0 is used (fixed position means velocity zero). When a system part needs to be excited, often a known signal form is needed, which can be modelled by a modulated source driven by some signal form. An example is shown in Figure 2.12.

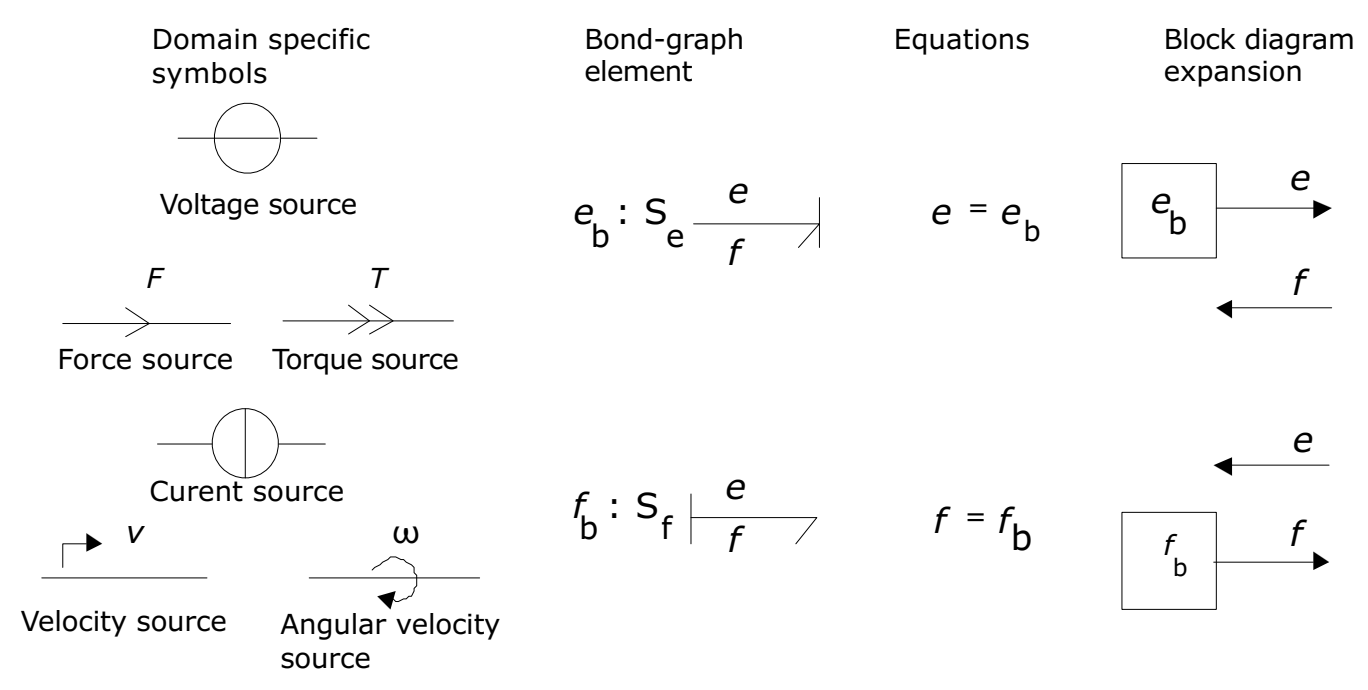

Fig. 2.11: Examples of sources

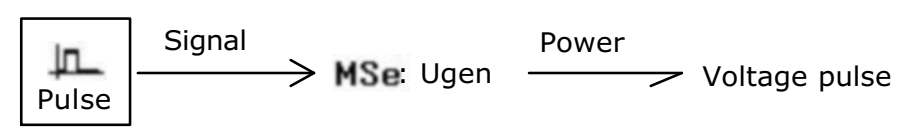

Fig. 2.12: Example of a modulated voltage source 


\subsubsection{Transformers and Gyrators}

An ideal transformer is represented by TF and is power continuous (i.e. no power is stored or dissipated). The transformation can within the same domain (toothed wheel, lever) or between different domains (electromotor, winch), see Figure 13. The equations are:

$$
\begin{aligned}
& e_{1}=n e_{2} \\
& f_{2}=n f_{1}
\end{aligned}
$$

Efforts are transduced to efforts and flows to flows. The parameter $\mathrm{n}$ is the transformer ratio. Due to the power continuity, only one dimensionless parameter, $n$, is needed to describe both the effort transduction and the flow transduction. The parameter $n$ is unambiguously defined as follows: $e_{1}$ and $f_{1}$ belong to the bond pointing towards the TF. This way of defining the transformation ratio is standard in leading publications [160],[44],[269],[67]. If $n$ is not constant, the transformer is a modulated transformer, a MTF. The transformer ratio now becomes an input signal to the MTF.

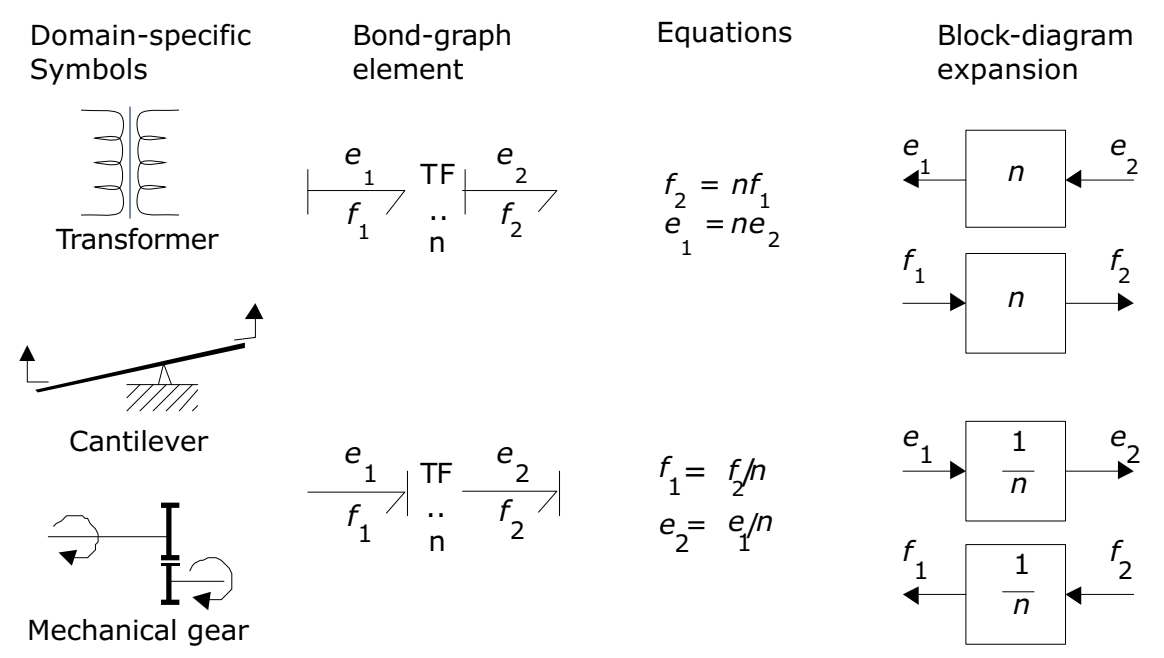

Fig. 2.13: Examples of transformers

An ideal gyrator is represented by GY, and is also power continuous (i.e. no power is stored or is dissipated. Examples are an electromotor, a pump and a turbine. Real-life realisations of gyrators are mostly transducers representing a domain-transformation (Figure 14). The equations are:

$$
\begin{aligned}
& e_{1}=r f_{2} \\
& e_{2}=r f_{1}
\end{aligned}
$$

The parameter $\mathrm{r}$ is the gyrator ratio, and due to the power continuity, only one parameter to describe both equations. No further definition is needed since the equations are symmetric (it does not matter which bond points inwards, only that one bond points towards and the other points form the gyrator). $r$ has a physical dimension, since $r$ is a relation between effort and flow (it has the same dimension as the parameter of the $\mathrm{R}$ element). If $r$ is not constant, the gyrator is a modulated gyrator, a MGY. 


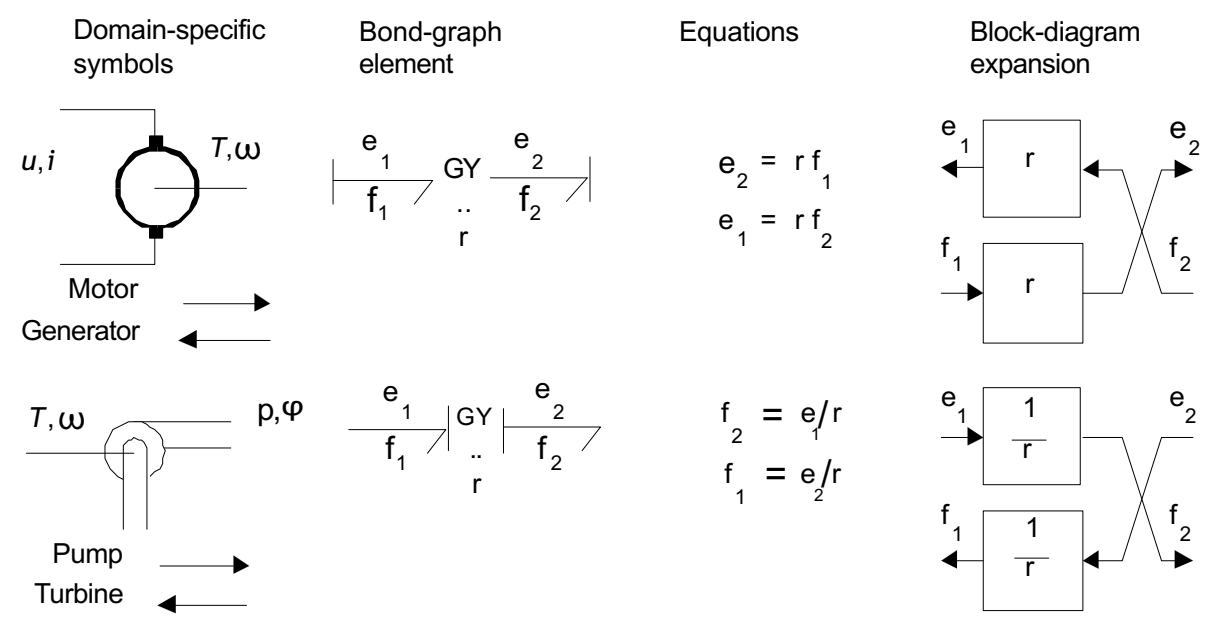

Fig. 2.14: Examples of gyrators

\subsubsection{Junctions}

Junctions couple two or more elements in a power continuous way: there is no energy storage or dissipation in a junction. Examples are a series connection or a parallel connection in an electrical network, a fixed coupling between parts of a mechanical system. Junctions are port-symmetric: the ports can be exchanged in the constitutive equations. Following these properties, it can be proven that there exist only two pairs of junctions: the 1 -junction and the 0 -junction.

The 0 -junction represents a node at which all efforts of the connecting bonds are equal (2.15). An example is a parallel connection in an electrical circuit. Due to the power continuity, the sum of the flows of the connecting bonds is zero, considering the sign. The power direction (i.e. direction of the half arrow) determines the sign of the flows: all inward pointing bonds get a plus and all outward pointing bonds get a minus. (Figure $\mathrm{X}$ ). This summation is the Kirchhoff current law in electrical networks: all currents connecting to one node sum to zero, considering their signs: all inward currents are positive and all outward currents are negative.

We can depict the 0 -junction as the representation of an effort variable, and often the 0 -junction will be interpreted as such. The 0-junction is more than the (generalised) Kirchhoff current law, namely also the equality of the efforts (like electrical voltages being equal at a parallel connection).

The 1 -junction (2.16) is the dual form of the 0 -junction (roles of effort and flow are exchanged). The 1 -junction represents a node at which all flows of the connecting bonds are equal. An example is a series connection in an electrical circuit. The efforts sum to zero, as a consequence of the power continuity. Again, the power direction (i.e. direction of the half arrow) determines the sign of the efforts: all inward pointing bonds get a plus and all outward pointing bonds get a minus. This summation is the Kirchhoff voltage law in electrical networks: the sum of all voltage differences along one closed loop (a mesh) is zero. In the mechanical domain, the 1-junction represents a force balance (also called the principle of d'Alembert), and is a generalisation of Newton's third law, action $=-$ reaction $)$.

Just as with the 0 -junction, the 1 -junction is more than these summations, namely the equality of the flows. Therefore, we can depict the 1-junction as the representation of a flow variable, and often the 1-junction will be interpreted as such.

\subsubsection{Positive Orientation}

By definition, the power is positive in the direction of the power bond (i.e. direction of the half arrow). A port that has an incoming bond connected to, consumes power if this power is positive (i.e. both effort and flow are 
Domain-specific symbols

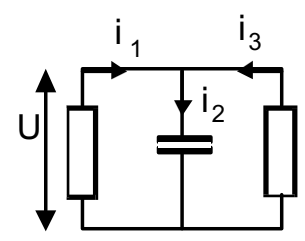

Bond-graph element

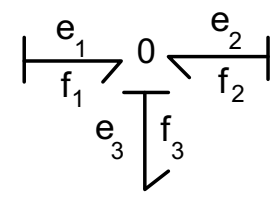

Equations

$$
\mathrm{e}_{1}=\mathrm{e}_{3}
$$$$
\mathrm{e}_{2}=e_{3}
$$$$
f_{3}=f_{1}-f_{2}
$$

Block-diagram expansion

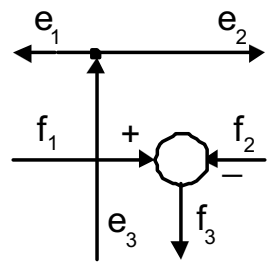

Fig. 2.15: Examples of gyrators

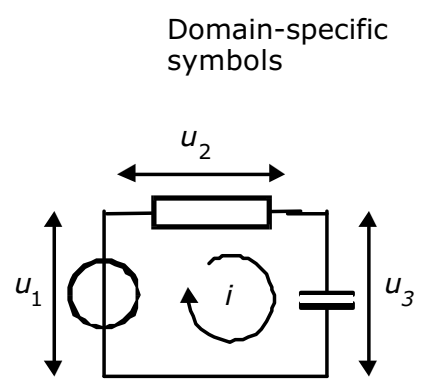

Bond-graph element

Equations

Block diagram expansion
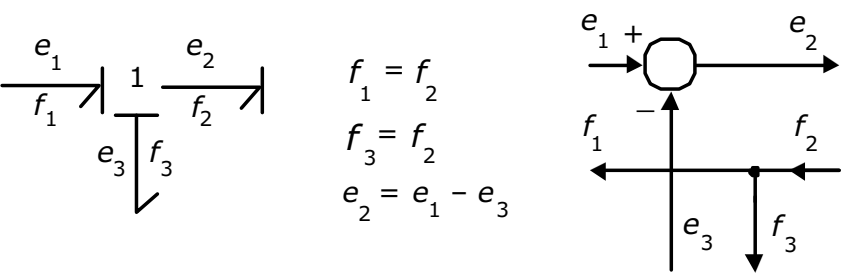

Fig. 2.16: Examples of gyrators

either positive or negative, as the product of effort and flow is the power). In other words: the power flows in the direction of the half arrow if it is positive and the other way if it is negative.

R-, C- and I-elements have an incoming bond (half arrow towards the element) as standard, which results in positive parameters when modelling real-life components. For source elements, the standard is outgoing, as sources mostly deliver power to the rest of the system. A real-life source then has a positive parameter. For TF- and GY-elements (transformers and gyrators), the standard is to have one bond incoming and one bond outgoing, to show the 'natural' flow of energy. Furthermore, using the standard definition of the parameter at the transformer (incoming bond is connected to port 1 and the ratio $n$ is $e_{1} / e_{2}$ ) positive parameters will be the result. Note that a gyrator does not need such a definition, since its equations are symmetric.

It is possible, however, that negative parameters occur. Namely, at transformers and sources in the mechanical domain when there is a reverse of velocity or the source acts in the negative direction.

Using the definitions discussed in this section, the bond-graph definition is unambiguous, implying that in principle there is no need for confusion. Furthermore, this systematic way will help resolving possible sign-placing problems often encountered in modelling, especially in mechanical systems. 


\subsubsection{Duality and Dual Domains}

As indicated in 2.4.1, the two storage elements are each other's dual form. The role of effort and flow in a C-element and I-element are exchanged. Leaving one of the storage elements (and also one of the sources) out of the list of bond graph elements, to make this list as small as possible, can be useful from a mathematical viewpoint, but does not enhance the insight in physics.

Decomposing an I-element into a GY and a C, though, gives more insight. The only storage element now is the C-element. The flow is only a time derivative of a conserved quantity, and the effort determines the equilibrium. This implies that the physical domains are actually pairs of two dual domains: in mechanics, we have potential and kinetic domains for both rotation and translation), in electrical networks, we have the electrical and magnetic domains. However, in the thermodynamic domain, no such dual form exists (Breedveld, 1982). This is consistent with the fact that no thermal I-type storage exists (as a consequence of the second law of thermodynamics: in a thermally isolated system, the entropy never decreases).

\subsection{Systematic Procedure to Derive a Bond-Graph Model}

In the previsous section, we have discussed the basic bond-graph elements and the bonds, so we can transform a domain-dependent ideal-physical model, written in domain-dependent symbols, into a bond graph. For this transformation, there is a systematic procedure, which will be presented in the next section.

To generate a bond-graph model starting from an ideal-physical model, a systematic method exist, which we will present here as a procedure. This procedure consists roughly of the identification of the domains and basic elements, the generation of the connection structure (called the junction structure), the placement of the elements, and possibly simplifying the graph. The procedure is different for the mechanical domain compared to the other domains. These differences are indicated between parenthesis. The reason is that elements need to be connected to difference variables or across variables. The efforts in the non-mechanical domains and the velocities (flows) in the mechanical domains are the across variables we need.

\subsubsection{The Eight Steps of the Systematic Procedure}

Steps 1 and 2 concern the identification of the domains and elements.

1. Determine which physical domains exist in the system and identify all basic elements like C, I, R, Se, Sf, $\mathrm{TF}$ and GY. Give every element a unique name to distinguish them from each other.

2. Indicate in the ideal-physical model per domain a reference effort (reference velocity with positive direction for the mechanical domains).

Note that only the references in the mechanical domains have a direction.

Steps 3 through 6 describe the generation of the connection structure (called the junction structure).

3. Identify all other efforts (mechanical domains: velocities) and give them unique names.

4. Draw these efforts (mechanical: velocities), and not the references, graphically by 0 -junctions (mechanical: 1 -junctions). Keep if possible, the same layout as the IPM.

5. Identify all effort differences (mechanical: velocity ( $=$ flow) differences) needed to connect the ports of all elements enumerated in step 1 to the junction structure. Give these differences a unique name, preferably showing the difference nature. The difference between $e_{1}$ and $e_{2}$ can be indicated by $e_{1} 2$.

6. Construct the effort differences using a 1 -junction (mechanical: flow differences with a 0 -junction) according to Figure 2.17, and draw them as such in the graph.

The junction structure is now ready and the elements can be connected.

7. Connect the port of all elements found at step 1 with the 0 -junctions of the corresponding efforts or effort differences (mechanical: 1-junctions of the corresponding flows or flow differences).

8. Simplify the resulting graph by applying the following simplification rules (2.18): 

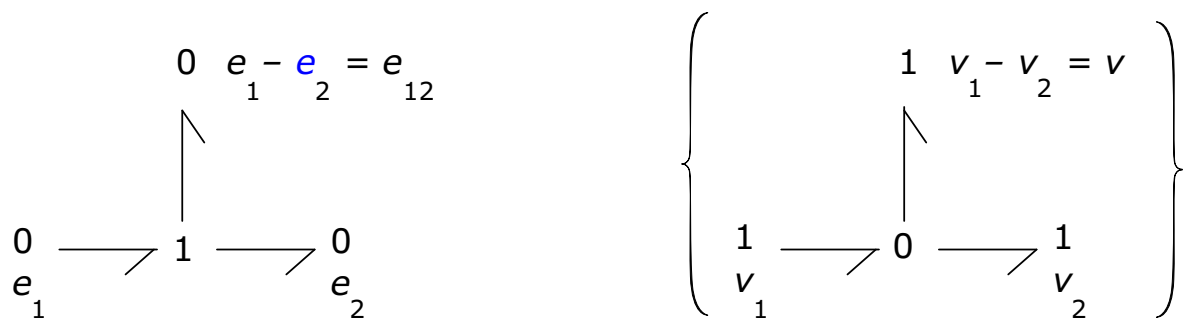

Fig. 2.17: Construction of effort differences (flow differences)

- A junction between two bonds can be left out, if the bonds have a 'through' power direction (one bond incoming, the other outgoing).

- A bond between two the same junctions can be left out, and the junctions can join into one junction.

- Two separately constructed identical effort or flow differences can join into one effort or flow difference.
a $\frac{e_{\text {in }}}{f_{\text {in }}}>0 \frac{e_{\text {uit }}}{f_{\text {uit }}} \quad=\frac{e}{f}$
b $\frac{e_{\text {in }}}{f_{\text {in }}}>1 \frac{e_{\text {uit }}}{f_{\text {uit }}} \quad=\frac{e}{f}$



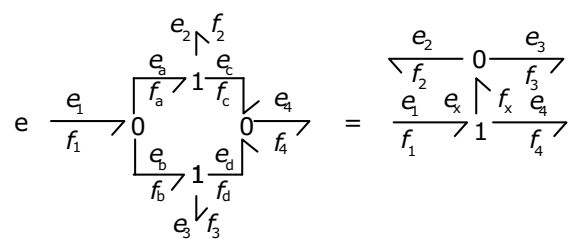

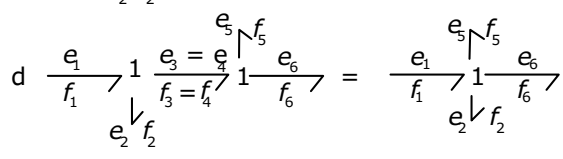

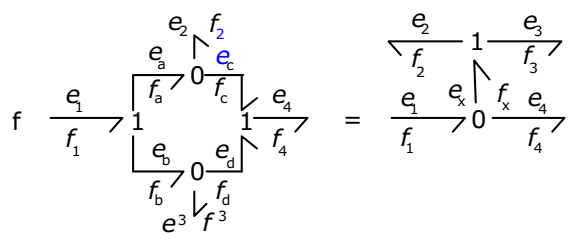

Fig. 2.18: Simplification rules for the junction structure: (a, b): elimination of a junction between bonds; (c, d): contraction of two the same junctions; $(e, f)$ : two separately constructed identical differences fuse to one difference.

\subsubsection{Illustration of the Systematic Procedure}

We will illustrate these steps with a concrete example consisting of an electromotor fed by electric mains, a cable drum and a load (2.19).

A possible ideal-physical model (IPM) is given in Figure 2.20. The mains is modelled as an ideal voltage source. At the electromotor, the inductance, electric resistance of the coils, bearing friction and rotary inertia are taken into account. The cable drum is the transformation from rotation to translation, which we consider as ideal. The load consists of a mass and the gravity force. Starting from the IPM of Figure 2.20, we will construct a bond graph using the 8 steps mentioned above. 


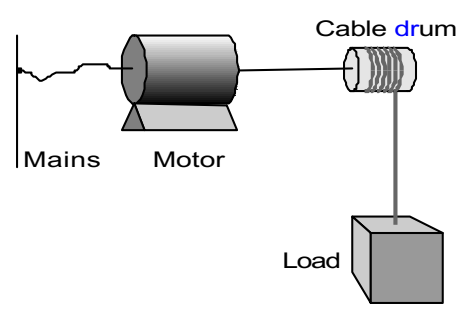

Fig. 2.19: Sketch of the hoisting device

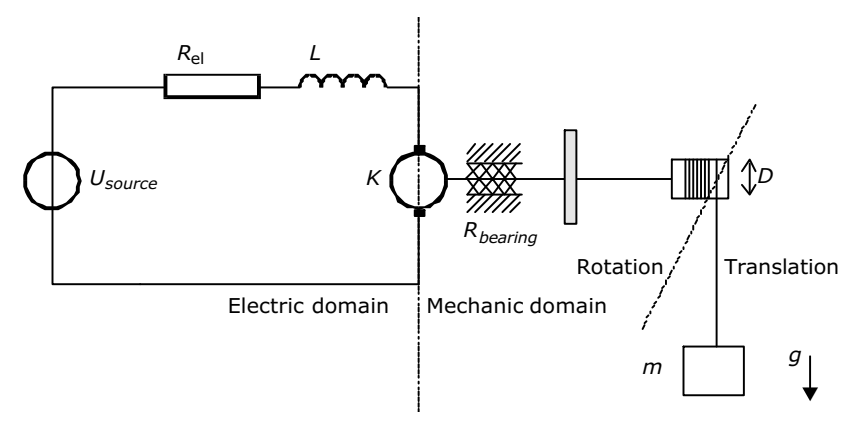

Fig. 2.20: Possible ideal-physical model augmented with the domain information of step 1

\section{Step 1}

This system contains:

- An electric domain part with a voltage source (Se), a resistor (R), an inductor (I) and the electric port of the electromotor (GY port).

- A rotation mechanic domain part with the rotation port of the electromotor (GY port), bearing friction (R), inertia (I), and the axis of the cable drum (TF port).

- A translation mechanic domain part with the cable of the cable drum (TF port), the mass of the load (I) and the gravity force acting on the mass (Se).

In Figure 2.20, the domains are indicated and all elements have a unique name.

\section{Step 2}

The references are indicated in the ideal physical model: the voltage $u_{0}$, the rotational velocity $\omega_{0}$ and the linear velocity $v_{0}$. The two velocities also get a positive orientation (i.e. a direction in which the velocity is positive). This result is shown in Figure 2.21.

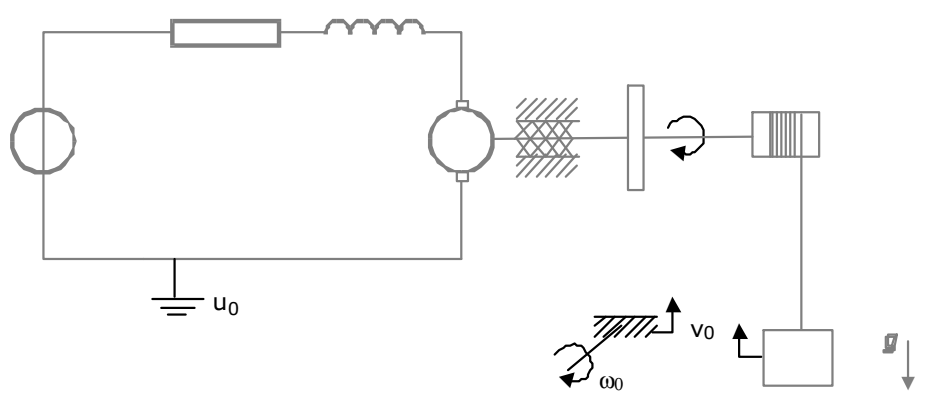

Fig. 2.21: References added to the IPM. 


\section{Step 3}

The other voltages, angular velocities and linear velocities are sought for and are indicated in the IPM (2.22). These variables are respectively $u_{1}, u_{2}, u_{3}, \omega_{1}, v_{1}$.

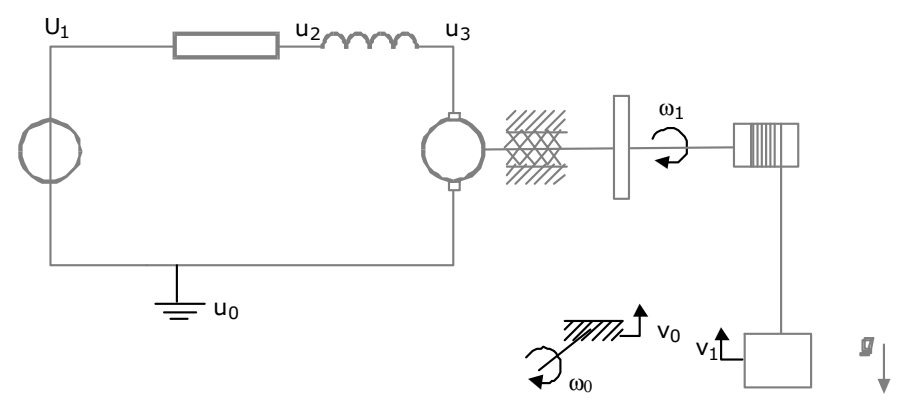

Fig. 2.22: The IPM augmented with relevant voltages, velocities, and angular velocities.

\section{Step 4}

The variables found in step 3 are depicted with 0 - respectively 1-junctions in Figure 2.23, in a layout compatible to the IPM. The references are not drawn, because they are so to speak eliminated (references have the value 0 and do not contribute to the dynamic behaviour).

$\begin{array}{llll}\stackrel{0}{\mathrm{u}_{1}} & \mathrm{O}_{2} & \mathrm{O}_{3} & 1 \\ \mathrm{u}_{1} & & \end{array}$

Fig. 2.23: First skeleton of the bond graph: Voltages are shown as 0 -junctions and velocities as 1 -junctions.

\section{Step 5}

When checking all ports of the elements found in step 1 for voltage differences, angular velocity differences and linear velocity differences, only $u_{1} 2$ and $u_{2} 3$ are identified. No velocity differences are needed.

\section{Step 6}

The difference variables are drawn in the bond graph, see Figure 2.24. After this step, the junction structure is generated and the elements can be connected.

\section{Step 7}

All elements are connected to the appropriate junctions, as shown in Figure 2.25. Note that non-mechanical domain elements are always connected to 0 -junctions (efforts or effort differences) and that mechanical domain elements are always connected to 1 -junctions.

\section{Step 8}

As last action, the bond graph needs to be simplified, to eliminate superfluous junctions (according to the rules given in Figure 2.18). The resulting bond graph is the outcome of the systematic method, see Figure 2.26.

Obviously, this systematic method is not the only method for deriving bond graphs from ideal physical models (IPMs). Another method is the so-called inspection method, where parts of the IPM are recognised that can be 




Fig. 2.24: Junction Structure ready: Difference variables $\left(u_{12}\right.$ and $\left.u_{23}\right)$ shown in the bond graph.

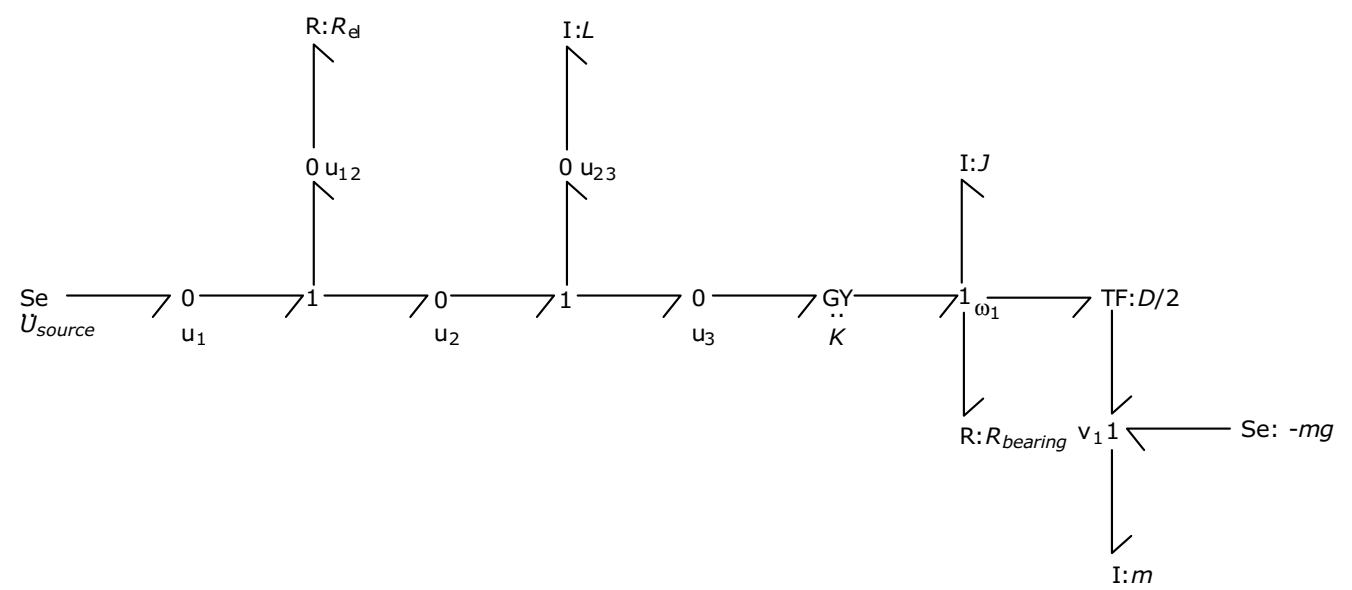

Fig. 2.25: The IPM augmented with relevant voltages, velocities, and angular velocities.

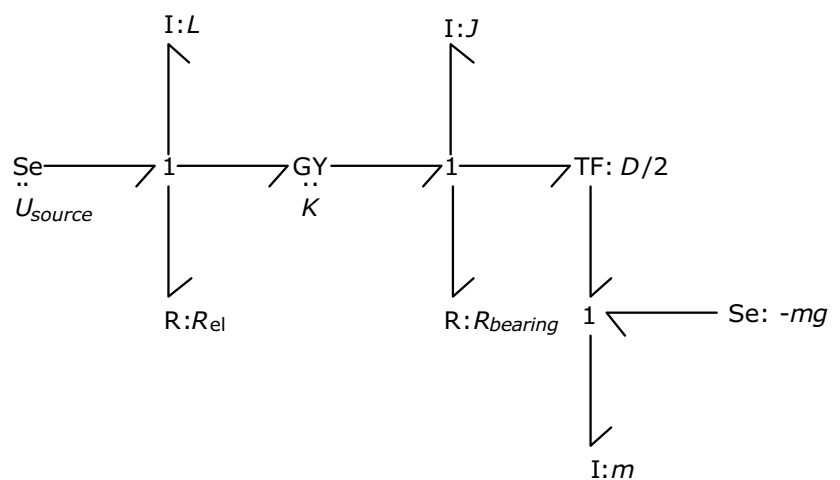

Fig. 2.26: The simplified bond graph, the result of the systematic method.

represented by one junction. An example is a series connection in an electrical network, which is drawn as one 1 -junction. This is the case in the example above: The voltage source, inductor, electric resistor and electric port of the motor are directly connected to one 1-junction. Although the inspection method is shorter than the systematic method, it is rather error prone. 


\subsection{Causal Analysis}

Causal analysis is the determination of the signal direction of the bonds. The energetic connection (bond) is now interpreted as a bi-directional signal flow. The result is a causal bond graph, which can be seen as a compact block diagram. Causal analysis is in general completely covered by modelling and simulation software packages that support bond graphs like Enport [242], MS1 [191], CAMP [129] and 20-sim [46, 48, 49, 50]. Therefore, in practice, causal analysis need not be done by hand. Besides derivation of equations, causal analysis can give insight in the correctness and competency of the model. This last reason especially motivates the discussion of causal analysis in this chapter.

\subsubsection{Causal Constraints}

Dependent on the kind of equations of the elements, the element ports can impose constraints on the connected bonds. There are four different constraints, which will be treated before a systematic procedure for causal analysis of bond graphs is discussed.

\subsubsection{Fixed causality}

Fixed causality is the case, when the equations only allow one of the two port variables to be the outgoing variable. This occurs at sources: an effort source (Se) has by definition always its effort variable as signal output, and has the causal stroke outwards. This causality is called effort-out causality or effort causality. A flow source (Sf) clearly has a flow-out causality or flow causality.

Another situation where fixed causality occurs is at nonlinear elements, where the equations for that port cannot be inverted (for example, division by zero). This is possible at R, GY, TF, C and I elements. Thus, there are two reasons to impose a fixed causality:

1. There is no relation between the port variables.

2. The equations are not invertible ('singular').

\subsubsection{Constrained Causality}

At TF, GY, 0- and 1-junction, relations exist between the causalities of the different ports of the element. These relations are causal constraints, since the causality of a particular port imposes the causality of the other ports. At a TF, one of the ports has effort-out causality and the other has flow-out causality. At a GY, both ports have either effort-out causality or flow-out causality.

At a 0 -junction, where all efforts are the same, exactly one bond must bring in the effort. This implies that 0 -junctions always have exactly one causal stroke at the side of the junction. The causal condition at a 1 -junction is the dual form of the 0 -junction. All flows are equal, thus exactly one bond will bring in the flow, implying that exactly one bond has the causal stroke away from the 1 -junction.

\subsubsection{Preferred Causality}

At the storage elements, the causality determines whether an integration or differentiation with respect to time will be the case. Integration has preference above a differentiation. At the integrating form, an initial condition must be specified. Besides, integration with respect to time is a process, which can be realised physically. Numerical differentiation is not physically realisable, since information at future time points is needed. Another drawback of differentiation occurs when the input contains a step function: the output will then become infinite. Therefore, integrating causality is seen as the preferred causality. This implies that a C-element has effortout causality and an I-element has flow-out causality at its preference. These preferences are also illustrated in Figure 2.8 and Figure 2.9, when looking at the block-diagram expansion. 


\section{Effort-out vs. flow-out causality}

When a voltage $u$ is imposed on an electrical capacitor (a C-element), the current $i$ is the result of the constitutive equation of the capacitor:

$$
i=C \frac{\mathrm{d} u}{\mathrm{~d} t}
$$

A differentiation is thus happening. We have a problem when the voltage instantly steps to another value, since the current will be infinite (the derivative of a step is infinite). This is not the case when the current is imposed on a capacitor. Now, an integral is used:

$$
u=u_{0}+\int i \mathrm{~d} t
$$

The first case is flow-out causality (effort imposed, flow the result), and the second case is effort-out causality, which is the preferred causality. Furthermore, an effort-out causality also results in a state variable with initial condition $u_{0}$. At an inductor, the dual form of the C-element is the case: flow-out causality will result in an integral causality, being the preference.

\subsubsection{Indifferent Causality}

Indifferent causality is used, when there are no causal constraints! At a linear R, it does not matter which of the port variables is the output. Consider an electrical resistor. Imposing a current (flow) yields:

$$
u=R i
$$

It is also possible to impose a voltage (effort) on the linear resistor:

$$
i=\frac{u}{R}
$$

There is no difference choosing the current as incoming variable and the voltage as outgoing variable, or the other way around.

\subsubsection{Causal Analysis Procedure}

In terms of causal constraints, we can say that the Se and Sf have a fixed causality, the C and I have a preferred causality, the TF, GY, 0 and 1 have constrained causality, and the R has an indifferent causality (provided that the equations of these basic elements all are invertible). These causal forms have been shown in 2.4. When the equations are not invertible, a fixed causality must be used.

The procedure for assigning causality on a bond graph starts with those elements that have the strongest causality constraint namely fixed causality (deviation of the causality condition cannot be granted by rewriting the equations, since rewriting is not possible). Via the bonds (i.e. connections) in the graph, one causality assignment can cause other causalities to be assigned. This effect is called causality propagation: after one assignment, the causality propagates through the bond graph due to the causal constraints.

The causality assignment algorithm is as follows:

1a Chose a fixed causality of a source element, assign its causality, and propagate this assignment through the graph using the causal constraints. Go on until all sources have their causalities assigned.

$1 \mathrm{~b}$ Chose a not yet causal port with fixed causality (non-invertible equations), assign its causality, and propagate this assignment through the graph using the causal constraints. Go on until all ports with fixed causality have their causalities assigned.

2 Chose a not yet causal port with preferred causality (storage elements), assign its causality, and propagate this assignment through the graph using the causal constraints. Go on until all ports with preferred causality have their causalities assigned. 
3 Chose a not yet causal port with indifferent causality, assign its causality, and propagate this assignment through the graph using the causal constraints. Go on until all ports with indifferent causality have their causalities assigned.

Often, the bond graph is completely causal after step 2, without any causal conflict (all causal conditions are satisfied). If this is not the case, then the moment in the procedure where a conflict occurs or where the graph becomes completely causal, can give insight in the correctness and competence of the model. Before discussing these issues, first an example will be treated.

\section{Causality algorithm}

To exemplify the causality algorithm, the same example as in 2.5 is used. In Figure 2.27, the completely causal bond graph of the hoisting device is shown. Numbers at the causal strokes indicate the order in which the bonds were made causal.

At step 1a, we assign causality 1 and 2 . It does not matter with which source we start. No causality can be propagated. Step $1 \mathrm{~b}$ is not applicable, since there are no ports of that category. At step 2, we started with the inductor. Propagation is from stroke 3 until stroke 6. The next storage element (preferred causality) is inertia J. Propagation of this causality (number 7), completes the causality of the graph, implying that we do not need step 3. However, the mass of the load does not get his preference. What the consequences are, is subject of the next section.

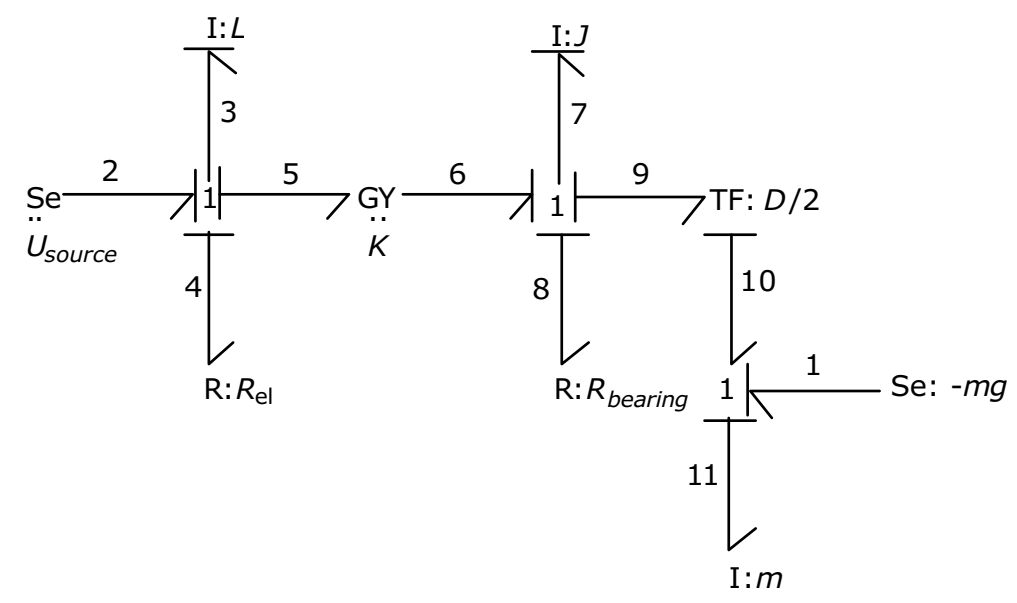

Fig. 2.27: The causal bond graph of the hoisting device.

\subsubsection{Model Insight via Causal Analysis}

We discuss here those situations whereby conflicts occur in the causal analysis procedure or when step 3 of the algorithm appears to be necessary. The place in procedure where a conflict appears or the bond graph becomes completely causally augmented, can give insight in the correctness of the model.

Often, the bond graph is completely causal after step 2, without any causal conflict (all causal conditions are satisfied). Each storage element represents a state variable, and the set of equations is an explicit set of ordinary differential equations (not necessarily linear or time invariant).

When the bond graph is completely causal after step $1 a$, the model does not have any dynamics. The behaviour of all variables now is determined by the fixed causalities of the sources. Arises a causal conflict at step 1a or at step $1 \mathrm{~b}$, then the problem is ill posed. The model must be changed, by adding some elements. An example of 
a causal conflict at step 1a is two effort sources connected to one 0 -junction. Both sources 'want' to determine the one effort variable.

At a conflict at step $1 b$, a possible adjustment is changing the equations of the fixed-causality element such that these equations become invertible, and thus the fixedness of the constraint disappears. An example is a diode or a valve having zero current resp. flow while blocking. Allowing a small resistance during blocking, the equations become invertible.

When a conflict arises at step 2, a storage element receives a non-preferred causality. This means that this storage element does not represent a state variable. The initial value of this storage element cannot be chosen freely. Such a storage element often is called a dependent storage element. This indicates that a storage element was not taken into account during modelling, which should be there from physical systems viewpoint. It can be deliberately omitted, or it might be forgotten. At the hoisting device example, the load of the hoist (I-element) is such a dependent storage element. Elasticity in the cable was not modelled. If it had been modelled, a C-storage element connected to a 0 -junction between the cable drum and load would appear, and would take away the causal conflict.

When step 3 of the causality algorithm is necessary, a so-called algebraic loop is present in the graph. This loop causes the resulting set differential equations to be implicit. Often this is an indication that a storage element was not modelled, which should be there from a physical systems viewpoint.

In general, different ways to handle the causal conflicts arising at step 2 or step 3 are possible:

1. Add elements.

For example, you can withdraw the decision to neglect certain elements. The added elements can be parasitic, for example, to add elasticity (C-element) in a mechanical connection, which was modelled as rigid. Additionally adding a damping element $(\mathrm{R})$ reduces the simulation time considerably, which is being advised.

2. Change the bond graph such that the conflict disappears.

For a step- 2 conflict, the dependent storage element is taken together with an independent storage element, having integral causality. For a step-3 conflict, sometimes resistive elements can be taken together to eliminate the conflict. This can be performed via transformations in the graph. The complexity of this operation depends on the size and kind of submodels along the route between the storage elements or resistors under concern.

3. The bond graph is not changed.

For simulation a special (implicit) integration routine is needed. The implicit equations are computed using an iteration scheme, mostly as part of the numerical integration method.

Algebraic loops and loops between a dependent and an independent storage element are called zero-order causal paths (ZCPs). Besides these two kinds, there are three other kinds, having an increasing complexity and resulting in more complex equations. These occur for instance in rigid-body mechanical systems (van Dijk and Breedveld, 1991).

By interpreting the result of causal analysis, several properties of the model can be recognised, which could otherwise only be done after deriving equations.

\subsubsection{Order of the Set State Equations}

The causal analysis also gives information on the order of the set equations. The number of initial conditions equals the number of storage elements with integral causality, which was also the preference during causality assignment. This number is called the order of the system. In the example (2.28), the order of the system is 2 .

The order of the set state equations is smaller than or equal to the order of the system, because storage elements can depend on each other. These kind of dependent storage elements each have their own initial value, but they together represent one state variable. Their input signals are equal, or have a factor in between (2.28).

A recipe exists to check whether this kind of dependent storage elements show up: Perform causal analysis again, but chose differential causality as preference. For the example, this is done in Figure 2.29.

Those storage elements that get both at differential preference and at integral preference their preferred causality are the real storage elements and contribute to the state of the system. The order of the set of state equations, is by definition the amount of storage elements that get in both cases their preferred causality. At 

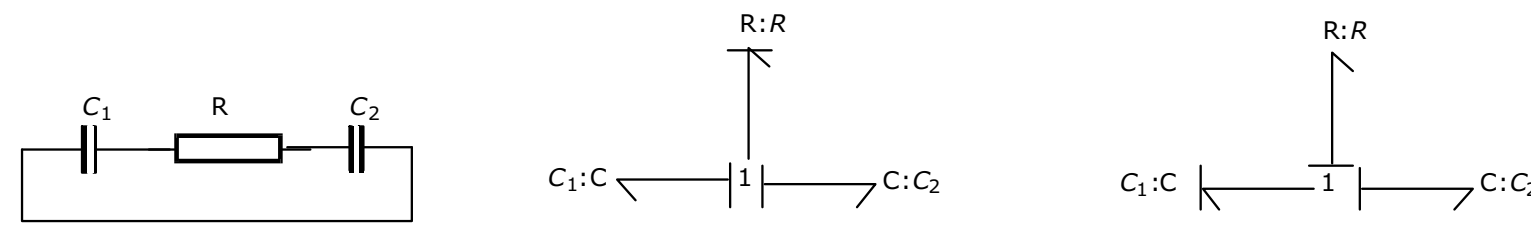

Fig. 2.28: System with order of state equations smaller than order of the system. a) IPM; b) causality using integral preference; c) causality using differential preference.



Fig. 2.29: Differential causality as preference applied to the hoisting device.

the hoisting device, this is 2 (namely $\mathrm{L}$ and $\mathrm{J}$ ). The storage elements that get in both cases not their preferred causality, are the dependent storage elements. Those storage elements that get only at the integral preferred causality case their preferred causality are called semi-dependent storage elements, to distinguish them from the dependent storage elements. This indicates that a storage element was not taken into account during modelling, which should be there from physical systems viewpoint.

\subsubsection{Matrix Form of Linear Systems}

If the system is linear, we can write the resulting set of state equations in the standard form, namely,

$$
\dot{\mathbf{x}}=\mathbf{A x}+\mathbf{B u}
$$

where $\mathbf{A}$ is the system matrix, and $\mathbf{A}$ is the input matrix. The order of the system is the dimension of the square matrix $\mathbf{A}$ and the order of the set of state equations is the rank of $\mathbf{A}$.

When dependent states or algebraic loops are present, the matrix description is as follows:

$$
\mathbf{E} \dot{\mathbf{x}}=\mathbf{A x}+\mathbf{B u}
$$

where $\mathbf{E}$ is a square matrix of size equal to the amount of storage elements plus the amount of algebraic loops. For each differential storage element and for each algebraic loop, $\mathbf{E}$ contains one row of zeros. The state vector $\mathbf{x}$ is extended with the algebraic loop variables, to enable implicit integration methods to solve these kinds of systems. The hoisting device gives such a set of equations. 


\subsection{Generation of Equations}

A causal bond graph contains all information to derive the set of state equations. It is either a set of ordinary first-order differential equations, ODEs, when the model is explicit (no causal conflicts), or a set of differential and algebraic equations, DAEs, when the model is implicit (a causal conflict in step 2 of the procedure or step 3 is necessary, cf. 2.6.2).

The procedure to derive the equations is covered by bond-graph software like Enport [242], MS1 [191], CAMP [129] and 20-sim [46, 48, 49, 50]. Therefore, in practice, generation of equations need not be done by hand. However, we do discuss the generation of equations on the one hand to be complete and on the other hand to indicate what exactly has to be done.

We use the following procedure to generate equations:

1. We first write the set of mixed differential and algebraic equations. These are the constitutive relations of all elements in computational form, or causal form. This comprises of $2 n$ equations of a bond graph having $n$ bonds. $n$ equations compute an effort and $n$ equations compute a flow, or derivatives of them.

2. We then eliminate the algebraic equations. We can organise this elimination process by first eliminate the identities coming from the sources and junctions. Thereafter, we substitute the multiplications with a parameter, stemming from resistors and transducers (TF, GY). At last, we substitute the summation equations of the junctions into the differential equations of the storage elements. Within this process, it is efficient to first mark the state variables. In principle, the state variables are the contents of the storage elements ( $p$ or $q$ type variables). However, if we write the constitutive relations of storage elements as one differential equation, we can also use the efforts at C-elements and flows at I-elements.

If we are going to generate the equations by hand, we can take the first elimination step into account while formulating the equations by, at the sources, directly use the signal function at the bond. Furthermore, we can write the variable determining the junction along all bonds connected to that junction. The variable determining the junction is that variable, which gets assigned to bond variables of all the other bonds connected to that junction via the identities of the junction equations. At a 0 -junction, this is the effort of the only bond with its causal stroke towards the 0 -junction. At a 1 -junction, this is the flow of the only bond with its causal stroke away from the 1-junction.

In case of dependent storage elements, we have to take care that the accompanying state variable gets not eliminated. These are the so-called semi state variables. When we mark the state variables, including the semi state variables in this situation, on beforehand, we can prevent the wrong variable from being eliminated. In case of algebraic loops, implicit equations will be encountered. We choose one of the variables in these loops as algebraic loop breaker and that variable becomes a semi state variable. See also 2.6.5. The equation consisting the semi state variable of a storage element gets eliminated at the second elimination step: it is a multiplication. The semi state variable itself must not be eliminated.

\section{Deriving the diferential equations of the hoisting device}

The set of mixed differential and algebraic equations of the hoisting device is shown below. The efforts and flows are numbered in the same order as their causality was assigned. We have 22 equations, of which 11 compute a flow and 11 compute an effort. Since we want to generate the set of equations as differential equations, we write at the storage elements in integral form the equations as differential equations and not as integral equations. This is called deferred integration, see Figure 2.30.

As state variables, we have $f_{3}$ and $f_{7}$, whereas $f_{11}$ is the semi state variable belonging to the mass having derivative causality (it is an I element). Note that the equation of the mass is written in causal form: $e_{11}$ is the output. After eliminating the identities at the junctions and sources, we have 12 equations. Note that the flows of the two effort sources $\left(f_{1}\right.$ and $\left.f_{2}\right)$ are not used elsewhere, so we leave them out.

After substitution of the multiplication with resistors and transducers, our set of equations reduces to the following 6 equations: 2 differential equations, 1 constraint equation, computing the semi state variable $f_{11}$ and 4 junction equations. Now, only state variables, junction variables and input variables are used.

After substitution of the summations at the junctions into the differential equations and the constraint equations, the result is shown in Figure 2.31

This system is a linear system, so we can write the equations in matrix form (according to the second form of 2.6.5), shown in Figure 2.32. 


$$
\begin{aligned}
& e_{2}=u_{\text {source }} \\
& \frac{\mathrm{d} f_{3}}{\mathrm{~d} t}=\frac{1}{L} e_{3} \\
& e_{4}=R_{e l} f_{4} \\
& f_{2}=f_{3} \\
& f_{4}=f_{3} \\
& f_{5}=f_{3} \\
& e_{3}=e_{2}-e_{4}-e_{5} \\
& e_{5}=K f_{6} \\
& e 6=K f_{5} \\
& \frac{\mathrm{d} f_{7}}{\mathrm{~d} t}=\frac{1}{J} e_{7} \\
& e_{8}=R_{\text {bearing }} f_{8} \\
& f_{6}=f_{7} \\
& f_{8}=f_{7} \\
& f_{9}=f_{7} \\
& e_{7}=e_{6}-e_{8}-e_{9} \\
& e_{9}=-\frac{D}{2} e_{10} \\
& f_{10}=-\frac{D}{2} f_{9} \\
& f_{1}=f_{10} \\
& f_{11}=f_{10} \\
& e_{10}=e_{11}-e_{1} \\
& e_{1}=-m g \\
& e_{11}=m \frac{\mathrm{d} f_{11}}{\mathrm{~d} t}
\end{aligned}
$$

Fig. 2.30: Equations of the hoisting device.

$$
\begin{aligned}
\frac{\mathrm{d} f_{3}}{\mathrm{~d} t} & =\frac{1}{L} u_{\text {source }}-\frac{R_{e l}}{L} f_{3}-\frac{K}{L} f_{7} \\
\frac{\mathrm{d} f_{7}}{\mathrm{~d} t} & =\frac{K}{J} f_{3}-\frac{R_{\text {bearing }}}{J} f_{7}+\frac{D}{2} m \frac{\mathrm{d} f_{11}}{\mathrm{~d} t}+\frac{D}{2} m g \\
f_{11} & =-\frac{D}{2} f_{7}
\end{aligned}
$$

Fig. 2.31: Differential and constraint equations of the hoisting device.

$$
\left(\begin{array}{ccc}
1 & 0 & 0 \\
0 & 1 & -\frac{D}{2} \\
0 & 0 & 0
\end{array}\right) \frac{d}{d t}\left(\begin{array}{l}
f_{3} \\
f_{7} \\
f_{11}
\end{array}\right)=\left(\begin{array}{ccc}
-\frac{R_{e l}}{L} & -\frac{K}{L} & 0 \\
\frac{K}{J} & -\frac{R_{\text {bearing }}}{J} & 0 \\
0 & \frac{D}{2} & 1
\end{array}\right)\left(\begin{array}{l}
f_{3} \\
f_{7} \\
f_{11}
\end{array}\right)+\left(\begin{array}{cc}
\frac{1}{L} & 0 \\
0 & \frac{D}{2} \\
0 & 0
\end{array}\right)\left(\begin{array}{c}
u_{\text {source }} \\
m g
\end{array}\right)
$$

Fig. 2.32: DAE of hoisting device in matrix form

When the model first was made explicit by adding elements, according to alternative 1 of 2.6 .3 , the causal bond graph and the equations are given below. We add the elasticity of the rope: A C-element connected to a 0 -junction is inserted on the bond between the TF of the cable drum and the 1-junction of the payload (see Figure 2.33). The efforts and flows are numbered in the same order as their causality was assigned. We have 26 equations, of which 13 compute a flow and 13 compute an effort. 




Fig. 2.33: Causal bond graph of hoisting device with elasticity added

As state variables, we have $f_{3}, f_{7}, e_{11}$ and $f_{13}$. So four independent state variables. Applying the procedure of deriving equations leads to the set of state equations written in matrix form shown in Figure 2.34.

$$
\frac{d}{d t}\left(\begin{array}{c}
f_{3} \\
f_{7} \\
e_{11} \\
f_{13}
\end{array}\right)=\left(\begin{array}{cccc}
-\frac{\operatorname{Re} l}{L} & -\frac{K}{L} & 0 & 0 \\
\frac{K}{J} & -\frac{R_{\text {bearing }}}{J} & -\frac{D}{2} & 0 \\
-\frac{D}{2 C_{\text {elasticity }}} & -\frac{1}{C_{\text {elasticity }}} & 0 & 0 \\
0 & 0 & \frac{1}{m} & 0
\end{array}\right)\left(\begin{array}{c}
f_{3} \\
f_{7} \\
e_{11} \\
f_{13}
\end{array}\right)+\left(\begin{array}{cc}
\frac{1}{L} & 0 \\
0 & 0 \\
0 & 0 \\
0 & -\frac{1}{m}
\end{array}\right)\left(\begin{array}{c}
u_{\text {source }} \\
m g
\end{array}\right)
$$

Fig. 2.34: ODE of hoisting device in matrix form

\subsection{Expansion to Block Diagrams}

To show that a causal bond graph is a compact block diagram, we treat in this section the expansion of a causal bond graph to a block diagram. Furthermore, a block diagram representation of a system might be more familiar than a bond graph representation. Thus this work might help understanding bond graphs.

The expansion of a causal bond graph into a block diagram consists of three steps:

1. Expand all bonds to bilateral signal flows (two signals with opposite directions). The causal stroke determines in which direction the effort flows. The bond graph elements can be encircled to connect the signals to.

2. Replace the bond-graph elements by their block-diagram representations (see 2.4). Deduce the signs of the summations of the junctions from the directions of the bond arrows (half arrows, see 2.4.5). Often, it is efficient to determine those signs after all bond-graph elements are written in block diagram form.

The block diagram is ready in principle.

3. Redraw the block diagram in standard form: all integrators in an ongoing stream (form left to right) and all other operations as feedback loops. Of course, this is not always possible. Blocks might be taken together. 
Since block diagrams represent mathematical operations, for which commutative and associative properties apply, these properties can be used to manipulate the block diagram such that the result looks appealing enough.

As an example, we show the block diagram of the hoisting device, using the three steps to construct the block diagram. In Figure 2.35 the result after step 2 is shown, and in Figure 2.36 the block diagram in standard form is presented. The block diagram of the model with elasticity is shown in Figure 2.37.

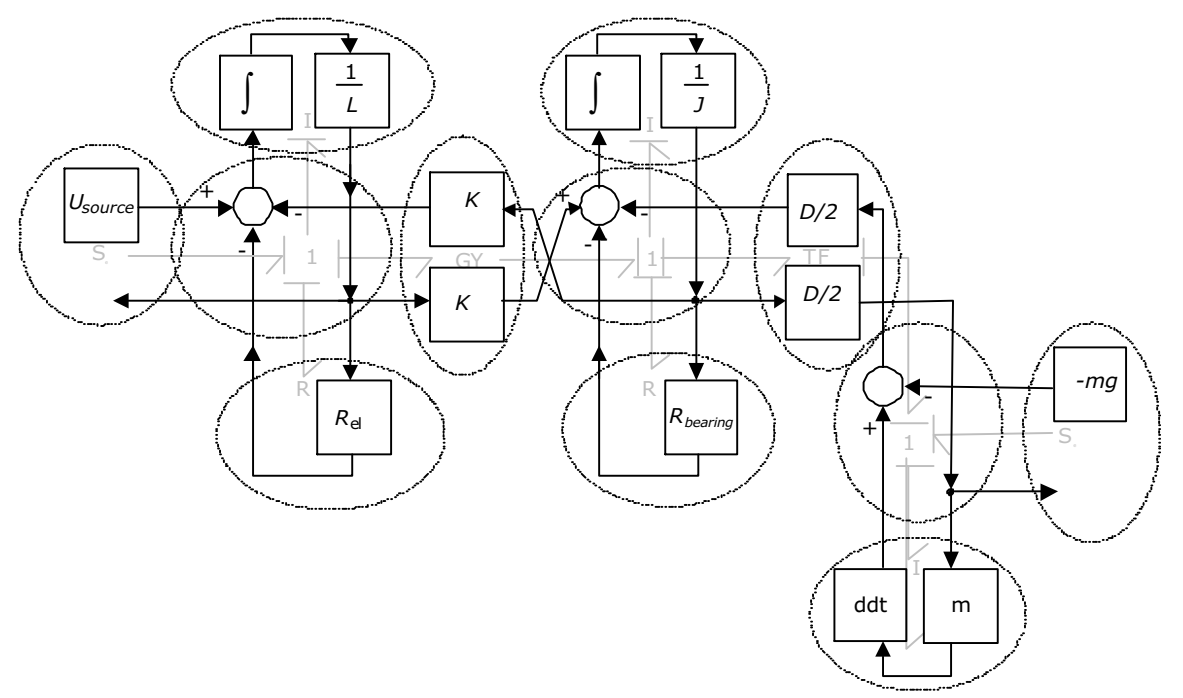

Fig. 2.35: Bond graph expanded to a block diagram in the layout of the bond graph (the bond graph is shown in grey).



Fig. 2.36: The block diagram redrawn in standard form.

\subsection{Simulation}

The resulting set of equations coming from a bond-graph model is called the simulation model. It consists of first-order ordinary differential equations (ODEs), possibly extended with algebraic constraint equations (DAEs). Hence, it can be simulated using standard numerical integration methods. However, because numerical integration is an approximation of the actual integration process, it is useful to check the simulation model on 


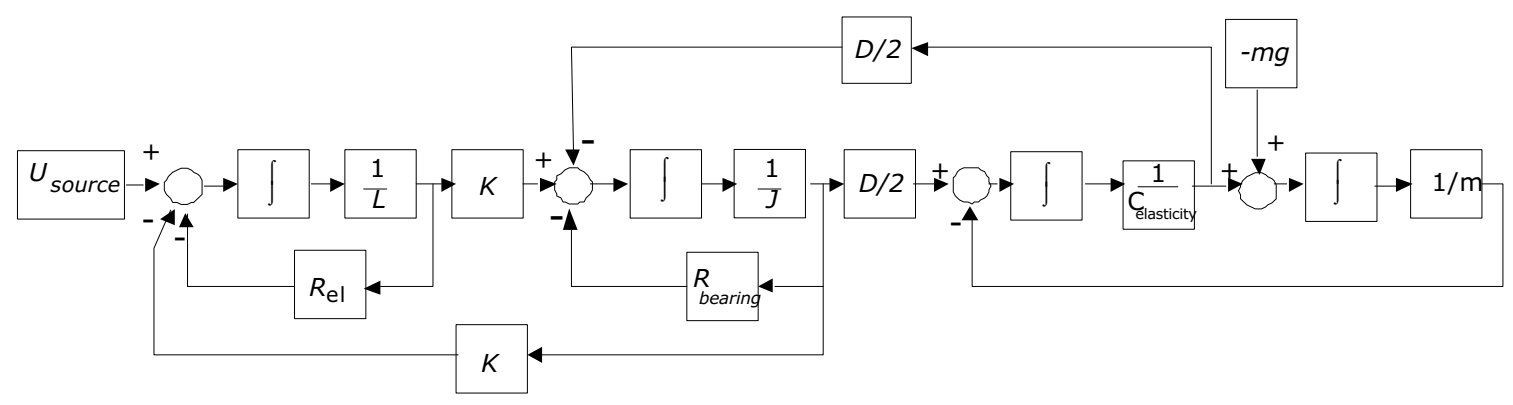

Fig. 2.37: The block diagram of the Hoist with elasticity

aspects significant for simulation. As a result, an appropriate integration method can be chosen: the computational work is minimal and the results stay within a specified error margin.

Since at causal analysis, one can decide whether or not to change the bond-graph model to obtain an explicit simulation model, it is useful to know about the consequences for simulation of relevant characteristics of the simulation model. The following 4 aspects of simulation models are relevant for choosing an numerical integration method:

1. Presence of implicit equations.

Implicit models (DAEs) can only be simulated with implicit integration methods. The iteration procedure of the implicit integration method is also used to calculate the implicit model. Explicit models (ODEs) can be simulated with both explicit as implicit integration methods. Sometimes, implicit integration methods need more computation time than explicit integration methods. During causal analysis, one can see whether a simulation model will be explicit or implicit, see 2.6.3.

2. Presence of discontinuities.

Integration methods with special provisions for events will perform best. If that is not available, variable step methods can be used. Multistep methods become less accurate, since they need information from the past, which is useless after a discontinuity. While constructing the model, presence of discontinuities can be marked.

3. Numerical stiffness.

$S(t)$, the stiffness ratio, is a measure for the distance between real parts of eigenvalues, $\lambda$, namely

$$
S(t)=\frac{\max (|\operatorname{Re} \lambda(t)|)}{\min (|\operatorname{Re} \lambda(t)|)}
$$

Stiff models (large $S$ ) need stiff integration methods. The time step is now determined by the stability instead of the accuracy (namely, eigenvalues are now used to determine the step size). When the high frequency parts are faded out, they do not influence the step size anymore. Hence, the step size can grow to limits determined by the lower frequencies.

4. Oscillatory parts.

When a model has no damping, it should not be simulated with a stiff method. Stiff methods perform badly for eigenvalues on the imaginary axis (i.e. no damping) of the complex eigenvalue plane.

Eigenvalues can be localised in a causal bond graph, especially when all elements are linear. There is a bond graph version of Mason's Loop rule to determine the transfer function from a bond graph [53]. As a side effect, the eigenvalues can be calculated. We will not discuss the procedure to obtain eigenvalues from a causal bond graph by hand. 


\subsection{Summary}

In this chapter, we have introduced bond graphs to model physical systems in a domain independent way. Only macroscopic systems are treated, thus quantum effects do not play a significant role. Domain independence has its basics in the fact that physical concepts are analogous for the different physical domains. Six different elementary concepts exist: storage of energy, dissipation, transduction to other domains, distribution, transport, input or output of energy.

Another starting point is that it is possible to write models as directed graphs: parts are interconnected by bonds, along which exchange of energy occurs. A bond represents the energy flow between the two connected submodels. This energy flow can be described as the product of 2 variables (effort and flow), letting a bond be conceived as a bilateral signal connection. During modelling, the first interpretation is used, while during analysis and equations generation the second interpretation is used.

Furthermore, we presented a method to systematically build a bond graph starting from an ideal physical model. Causal analysis gives, besides the computational direction of the signals at the bonds, also information about the correctness of the model. We presented methods to derive the causality of a bond graph. In addition, procedures to generate equations and block diagrams out of a causal bond graph are presented.

Due to the introductory nature of this text, some procedures presented, without a deep motivation and possible alternatives. It was also not the incentive to elaborate on physical systems modelling. We did not discuss multiple connections (arrays of bonds written as one multibond) and multiport elements (to describe transducers), neither different causal analysis algorithms. Those different causality algorithms give slightly different sets of DAEs especially when applied to certain classes of models (for instance multibody systems with kinematic loops).

Finally, bond-graph modelling is in fact a form of object-oriented physical-systems modelling, a term which is often used. This can be seen as follows: bond-graph models are declarative, they can be hierarchically structured, and fully support encapsulation (due to the non-causal way of specifying equations, and the notion of ports). Moreover, due to allowing hierarchy, the notion of definition and use of models are distinguished (i.e. the class concept and instantiation). Since bond graphs came into existence before the term object oriented was used in the field of physical systems modelling, bond graphs can be seen as an object-oriented physical-systems modelling paradigm avant-la-lettre.

A bond-graph library was written in Modelica, a contemporary object-oriented modelling language [47]. The basic bond-graph elements and block-diagram elements have been specified in Modelica, using the essential object-orientation features inheritance and encapsulation. Equations have been specified in an a-causal format. Thus, it can be said that the Modelica modelling concepts are consistent with bond-graph concepts. Furthermore, automatic Modelica code generation from bond graphs appeared to be rather straightforward [46].

\subsection{Literature and Further Reading}

For a more thorough analysis of bond graphs, see Paynter [228] and Breedveld [43, 44], while an extensive discussion on textbook level is given by Karnopp, Margolis and Rosenberg [160]. Cellier [67] wrote a textbook on continuous system modelling in which besides bond graphs also other modelling methods are used. Current research on bond graphs is reported at the International Conference on Bond Graph modelling, every two years (Granda and Cellier [129]). Journals regularly publishing bond graph papers are the Journal of the Franklin Institute, which also had special issues on bond graphs and the Journal of Dynamic Systems, Measurement and Control.

\subsection{Self-Assessment}

1. What is a bond? Why is starting with bonds as means of connecting subsystems beneficial and crucial?

2. What are conserved quantities? Give examples of each type of conserved quantity.

3. What is a causal constraint? Why is the order of using causal constraints in the causal analysis procedure as is?

4. What insight gives conducting the causal analysis procedure? 
5. What properties of a causal bond-graph model (or any model written as differential equations) influence the choice of the numerical integration algorithm needed for simulation of that model?

\section{Acknowledgements}

Most of the inspiration for this chapter came from the Dutch course material of Breedveld and Van Amerongen (1994). I sincerely acknowledge Peter Breedveld and Job van Amerongen for their valuable suggestions and discussions.

Open Access This chapter is licensed under the terms of the Creative Commons Attribution 4.0 International License (http://creativecommons.org/licenses/by/4.0/), which permits use, sharing, adaptation, distribution and reproduction in any medium or format, as long as you give appropriate credit to the original author(s) and the source, provide a link to the Creative Commons license and indicate if changes were made.

The images or other third party material in this chapter are included in the chapter's Creative Commons license, unless indicated otherwise in a credit line to the material. If material is not included in the chapter's Creative Commons license and your intended use is not permitted by statutory regulation or exceeds the permitted use, you will need to obtain permission directly from the copyright holder. 